Cinémas

Revue d'études cinématographiques

Journal of Film Studies

\title{
Intermédialité, deixis et politique
}

\section{Germain Lacasse}

Volume 10, numéro 2-3, printemps 2000

Cinéma et intermédialité

URI : https://id.erudit.org/iderudit/024817ar

DOI : https://doi.org/10.7202/024817ar

Aller au sommaire du numéro

Éditeur(s)

Cinémas

ISSN

1181-6945 (imprimé)

1705-6500 (numérique)

Découvrir la revue

Citer cet article

Lacasse, G. (2000). Intermédialité, deixis et politique. Cinémas, 10(2-3), 85-104. https://doi.org/10.7202/024817ar

\section{Résumé de l'article}

La sphère intermédiatique est un espace symbolique au sein duquel on peut s'approprier les récits par transfert médiatique. Leur deixis transformée, ces récits se voient reterritorialisés. Ce phénomène fut marquant au Québec au début du XXe siècle, alors qu'on adapta pour le vaudeville, le théâtre ou la radio locale nombre de récits du cinéma américain. d'utilisation que vous pouvez consulter en ligne.

https://apropos.erudit.org/fr/usagers/politique-dutilisation/ 


\section{Intermédialité, deixis et politique}

\section{Germain Lacasse}

\section{RÉSUMÉ}

La sphère intermédiatique est un espace symbolique au sein duquel on peut s'approprier les récits par transfert médiatique. Leur deixis transformée, ces récits se voient reterritorialisés. Ce phénomène fut marquant au Québec au début du $\mathrm{XX}^{\mathrm{c}}$ siècle, alors qu'on adapta pour le vaudeville, le théâtre ou la radio locale nombre de récits du cinérna américain.

\section{ABSTRACT}

The intermedial sphere is a symbolic space in which narratives are open to appropriation through a media transfer which results in the transformation of their deixis, as well as their reterritorialization. This was a significant phenomenon in Quebec where several forms of adaptation of American cinema narratives appeared in vaudeville, theater and local radio in the beginning of the twentieth century.

Alain Berrendonner, dans ses Éléments de pragmatique linguistique, appelle "fantôme de la vérité " l'instance universelle de validation des énoncés, qu'il définit aussi comme un "déictique de l'ordre des choses" (p. 59-60). Le spectre qu'il traque serait présent dans toutes les communications; celles-ci, plutôt que d'être dialogiques, constitueraient un jeu à trois termes: "Le plus remarquable me paraît être ici que l'univers, c'està-dire le "contexte" de tous les schémas de la communication, conçu comme entité référentielle globale, doive être considéré comme un participant actif de l'événement d'interlocution, et non seulement comme une circonstance inerte" (p. 61). Ces observations paraissent d'une grande justesse parce qu'elles 
concordent avec plusieurs exemples de capture et de transformation du fantôme, dont la place et la forme changent avec les vérités dont il serait l'invisible écho. Il se manifeste tout autant dans la sphère intermédiatique, où les énonciateurs essaient de le faire changer de position afin de valider leur propre vérité. Nous nous proposons donc ici de faire un peu de ghostbusting: traquer le spectre intermédiatique dans l'histoire et l'exorciser dans le présent. Nous commencerons cependant par proposer et analyser quelques définitions de cet univers spectral.

On pourrait appeler "sphère intermédiatique " ${ }^{1}$ l'espace à la fois réel et symbolique constitué par les médias et leur rapport avec les communautés. Empruntant partiellement la terminologie de Deleuze et Guattari dans Mille Plateaux, on pourrait aussi nommer "reterritorialisation virtuelle" le phénomène que constitue la reconfiguration du social dans l'intermédiatique. Deleuze et Guattari, fortement inspirés des écrits de Nietzsche sur les forces actives et sur les formes comme devenir ${ }^{2}$, décrivent d'abord l'existence de strates, qui sont des "phénomènes d'épaississement sur le Corps de la terre, à la fois moléculaires et molaires: accumulations, coagulations, sédimentations, plissements. [...] Chaque strate, ou articulation, consiste en milieux codés, substances formées" (1980, p. 627). Dans les strates et entre elles apparaissent les "agencements", qui sont d'abord territoriaux, et ces territoires sont faits de «[...] fragments décodés de toutes sortes, empruntés aux milieux, mais qui acquièrent alors une valeur de "propriétés" " (p. 629).

La territorialisation comporte contenu et expression, système sémiotique et système pragmatique, mais au niveau supérieur, l'agencement dont elle fait partie comporte lui aussi des «[...] lignes de déterritorialisation qui le traversent et l'emportent» (p. 630). La sphère intermédiatique serait donc un "agencement» où se succèdent la territorialisation sociale et la déterritorialisation symbolique. Le rapport de l'individu avec l'espace-temps réel y deviendrait un rapport entre le sujet et un espace-temps reconfiguré symboliquement. On pourrait citer comme exemples la Bible, récit de reterritorialisation symbolique de la communauté juive dans l'Antiquité, ou le cinéma québécois contemporain, affirmation dans le champ 
culturel d'une communauté longtemps brimée dans l'espace social.

Chacun de ces agencements suppose une deixis particulière, une configuration abstraite des rapports à l'espace, au temps et au social, puisque la deixis définit l'orientation du sujet dans l'espace et le temps. Les déictiques sont couramment définis comme les «termes servant à désigner». Wlad Godzich et Jeff Kittay, développant les thèses de Benveniste et Jakobson, démontrent que la deixis désigne également la contextualisation de l'énonciation, l'inscription du locuteur dans un espace-temps qui actualise et valide son discours (p. 18-23). L'apparition et l'évolution des médias supposent une évolution de cette structure abstraite. Les médias pourraient être considérés, ainsi que le proposait McLuhan, comme des prolongements des sens, mais chaque stade de leur évolution impliquerait une nouvelle symbolisation abstraite du rapport au monde qu'ils établissent et maintiennent. Cette symbolisation n'est évidemment plus structurée par des déictiques explicites, mais par cette deixis abstraite que Berrendonner appelle "fantôme de la vérité" et qu'Oswald Ducrot appelle la présupposition: "[...] acte par lequel le locuteur impose à un destinataire un certain univers de discours" (1972, p. 97). Cet acte se manifeste de différentes façons, mais n'affiche pas de marques aisément localisables. Pour les repérer dans l'univers médiatique, il faut d'abord comprendre la nature des médias et leur impact sur la deixis.

Après avoir approfondi la définition de deixis, il faut donc en faire autant pour média. Parmi les mots apparentés à média, on trouve médian: (lat. maedius, qui est au milieu) qui se trouve au milieu (Dictionnaire encyclopédique Larousse, 1994, p. 643). On trouve aussi médiat: qui a rapport, qui ne touche à une chose que par une autre; qui est intermédiaire (p. 643). Ce dernier terme nous conduit à médiatrice: droite perpendiculaire au segment en son milieu (p. 643). Quand nous parlons d'intermédialité, nous renvoyons donc à une notion qui fait apparaître un nœud de lignes clont chacune ne touche à une chose que par un point, mais où toutes les lignes se touchent. C'est forcé puisqu'elle peut être définie comme une multiplicité de lignes médianes. On peut aussi penser à une figure géométrique 
complexe à plusieurs dimensions, puisque plusieurs médiatrices s'y superposent et se croisent pour séparer et opposer différentes surfaces.

Il est facile de s'y perdre, et la seule façon de s'y retrouver est peut-être celle que suggérait Deleuze dans Dialogues: “ [...] faire la ligne, et pas le point" (Deleuze et Parnet, p. 34). Car faire le point est devenu impossible. Nous allons donc essayer de tracer la ligne de l'intermédialité, de la définir de façon diachronique, car nous croyons que la croissance fulgurante qu'elle a connue au cours des dernières décennies tend à faire oublier ses antécédents. Nous parlerons ici de préhistoire de l'intermédialité et d'intermédialité dans la préhistoire, cette hypothèse étant posée comme mode d'analyse paralogique d'un phénomène dont l'accélération semble actuellement effarante. Nous parlerons aussi encore de deixis, puisque la médialité renvoie à un sujet percevant et à sa position dans un monde, et nous proposerons d'envisager différentes configurations déictiques correspondant aux étapes historiques de l'intermédialité et coïncidant souvent avec l'histoire politique.

\section{La deixis pluritopique}

La perception sensorielle et sa mémorisation sont peut-être les premières opérations médiatiques; McLuhan disait que les médias sont les prolongements des sens (1972). Effectuons le parcours à rebours et voyons comment les sens correspondaient aux médias avant que ceux-ci n'apparaissent. La perception dans l'espace et le temps dépendait des organes sensitifs; l'information, dont on sait aujourd'hui qu'elle circule même entre les cellules, était également échangée au moyen des organes : voix et ouie, membres et vision, etc. Cette mémoire pourrait être considérée comme une opération intermédiatique consistant en l'encryptage biologique de la perception spatio-temporelle. La position du sujet dans l'espace reposait en quelque sorte sur une deixis sensorielle plutôt que discursive.

Cette deixis est bouleversée plutôt radicalement à mesure qu'évoluent le langage gestuel et le langage verbal. Leur capacité de transmission à distance et de conservation est indissociablement liée à l'expansion territoriale. Celle-ci provoque de 
nouveaux récits de mémorisation où le sujet est mis en relation avec des espaces distants, familiers ou étrangers, supposant une deixis maintenant manifestée dans le langage, et que nous qualifions de pluritopique puisqu'elle repose sur la relation d'un sujet avec plusieurs territoires. Faire la ligne devient déjà plus pertinent que de faire le point: quels sont les liens associant le sujet avec cet environnement étendu mais discontinu et fragmenté? Ces liens abstraits correspondent historiquement aux premiers arts intermédiatiques, les peintures des grottes pariétales dont l'anthropologue Leroi-Gourhan a souligné le " [...] contexte oral avec lequel l'assemblage symbolique était coordonné et dont il reproduit spatialement les valeurs» (p. 273).

L'occupation de ces territoires pluritopiques suppose non seulement une symbolisation différente et un niveau supérieur d'abstraction, mais aussi des moyens de communication à distance entre les membres de la communauté. D'où l'émergence de nouvelles pratiques signifiantes, émergence dont l'histoire fournit beaucoup d'exemples de l'émergence nouvelle. Godzich et Kittay ont montré de façon fort pertinente que la disparition de la poésie orale, ou du moins sa marginalisation, est liée à l'imposition et à la généralisation de la prose écrite par les groupes qui ont tiré profit de l'émergence de l'État vers la fin du Moyen Âge (p. 200). La généralisation de la prose écrite a imposé une nouvelle deixis, dont le degré d'abstraction s'est encore intensifié au moment de l'expansion territoriale, et aussi lorsque la hiérarchie s'est complexifiée: l'espace pluritopique correspond aussi à une centralisation de l'autorité, et cette centralisation ne semble jamais devoir s'arrêter. Lorsqu'elle est détruite, on la voit rapidement réapparaître sous une autre forme, s'efforçant de correspondre au "fantôme de la vérité " pour mieux convaincre ses interlocuteurs.

\section{Le cinéma oral}

Le cinéma oral est l'une de ces pratiques intermédiatiques qui résultent de l'apparition d'un nouveau média, de son institutionnalisation et des résistances qu'il suscite. À ses débuts, le cinéma tenait plus de l'attraction foraine que du média; sa capacité discursive était faible et il devait compter avec l'aide 
d'un explicateur verbal, ce dernier assurant la compréhension des éléments narratifs ${ }^{3}$. Le nouveau média devait faire appel à une ancienne pratique, et ce n'est qu'au bout d'un long travail de légitimation et de hiérarchisation qu'il est arrivé à s'en dégager. Le "cinéma muet» est devenu la pratique signifiante dominante dans les pays produisant des films, tandis que dans les communautés marginales et les colonies, le cinéma oral a eu cours très longtemps, dans certains cas jusqu'au milieu du siècle.

La Première Guerre mondiale semble marquer le triomphe du langage cinématographique institutionnalisé. Le film que l'on considère comme son modèle achevé, Birth of a Nation (Griffith), est sorti en 1916. Cette coïncidence n'est pas complètement fortuite; le processus de normalisation de la pratique était déjà enclenché, mais la guerre a poussé les États à accroître de façon totalitaire le contrôle des communications et des discours. Les bonimenteurs de films, dont le nombre avait diminué à mesure que le cinéma acquérait une capacité narrative, ont été rappelés en masse en 1914 pour commenter des films de guerre qui risquaient d'effrayer les civils, et pour attiser l'ardeur patriotique. Le gouvernement du Canada, qui ne s'était jamais préoccupé de cinéma, mit sur pied un service de propagande cinématographique dont la direction fut confiée à l'un de ses plus riches et puissants citoyens. Pourtant, c'est au même moment que semble s'être consolidée au Québec la pratique du cinéma oral, puisqu'il y est apparu alors ce qu'on appelle le burlesque québécois: un spectacle composé de danse, de thêâtre populaire comique et de vues animées bonimentées. On voit donc émerger presque simultanément deux pratiques intermédiatiques dont les discours divergent: celle d'un État propageant le cinéma oral comme moyen de soutenir son intervention armée, et celle d'une communauté consolidant le cinéma oral comme moyen de résistance.

La mémoire étant inscrite dans l'espace autant que dans le temps, les images animées constituent un outil providentiel pour un État en émergence qui veut suggérer à ses sujets une nouvelle représentation et une nouvelle assimilation de l'espace politique et de la temporalité historique. C'est donc du point de vue de leur deixis quil faut tenter de lire ces images pour comprendre 
comment l'État voulait s'y inscrire et s'y perpétuer. C'est par une deixis de l'absence qu'il le fait, par l'instauration d'un système de signes renvoyant à une autorité invisible et bureaucratique qui se manifeste en montrant qu'elle efface ses traces, en montrant qu'elle retient ses mots et peut-être son souffle... Les films de guerre nous permettent donc de lire comme par transparence les traces de la présence de l'État canadien: il n'est pas visible sur l'écran, mais très apparent derrière; transparent, il épouse la forme diffuse et mouvante du "fantôme de la vérité ».

Le résultat de cette longue campagne de propagande est l'émergence d'une deixis cinématographique endogène qui supplante la configuration exogène précédente. Le sujet canadien, auparavant spectateur accessoire de films étrangers, est devenu le partenaire des acteurs de l'intervention militaire. Pour maintenir ce nouveau point de vue, l'État canadien a d'ailleurs mis sur pied à la fin de la guerre le Canadian Government Motion Picture Bureau en lui confiant un mandat clair: offrir une image canadienne du Canada, aux Canadiens et aux étrangers; offrir aux citoyens un cadre montrant les fragments de l'espace pluritopique désormais occupé, un cadre permettant aussi d'exporter au loin les portions de l'espace canadien. Comme l'a souligné Deleuze, l'espace cinématographique est venu supplanter l'espace naturel et provoquer la dislocation des liens sensorimoteurs ${ }^{4}$. L'État en émergence trouvait ainsi moyen d'apparaître dans la nouvelle sphère et d'être inscrit dans l'univers qu'elle désignait. Il capturait le "fantôme de la vérité " et tentait de le garder captif derrière la trame de l'écran. L'écoute actuelle de ces films d'époque constitue d'ailleurs une expérience extrêmement étrange où la vérité est pour le moins spectrale. Voici le propos authentique d'un conférencier militaire présentant des films à un auditoire montréalais en 1916:

Toujours au grand air, la vie d'un soldat est saine et réconfortante. Le conférencier a rencontré d'anciens employés de bureaux qui traînaient jadis une santé chancelante et sont aujourd'hui plus forts et plus virils qu'ils ne l'ont jamais été après un an ou six mois de vie dans les tranchées. La nourriture est bonne et saine et on a le grand air continuellement. Rien de meilleur pour rendre à un homme la vitalité éteinte et perdue 5 . 
La perpétuation du cinéma oral comme pratique de résistance permettait par contre aux communautés résistantes de produire sur place leur discours d'appropriation et donc d'imposer à leur tour une autre deixis endogène. Le discours de l'État sur la guerre était atténué ou critiqué par les interventions des bonimenteurs, monologuistes, chanteurs et comédiens des communautés opposées à la guerre, dont les propos antibellicistes sont devenus l'une des hantises du fantôme de la vérité payé par l'État, le chef-censeur Ernest J. Chambers. Il n'est pas établi que le bonimenteur populaire se livrait à un commentaire critique des films de propagande, mais l'examen des traces sonores des salles où il exerçait montre un contexte fortement résistant et laisse penser qu'il a pu participer à une lecture résistante des films de guerre. Comme le conférencier militaire, le bonimenteur populaire semble avoir connu un regain de popularité pendant la guerre, dans une forme de spectacle qui s'est développé durant cette période au Québec. Variante du vaudeville américain, le burlesque québécois était constitué de numéros de danse, de sketches, de monologues comiques et de vues animées bonimentées (Hébert, 1981). L’opposition des Canadiens français à la guerre a stimulé le sentiment nationaliste, et les historiens du burlesque soulignent la volonté des artistes d'accentuer l'usage du français dans les spectacles. Plusieurs des bonimenteurs français qui pratiquaient leur métier sur les scènes du Québec sont repartis en France pour s'enrôler et ont cédé la place à des collègues québécois. Les échos des spectacles de l'époque sont devenus bien différents de ceux des soirées animées par un conférencier militaire.

La pièce de résistance à cet égard est certainement le monologue écrit en 1917 par Armand Leclaire, qui s'intitule simplement «Le conscrit Baptiste». Il raconte les bévues commises par un campagnard québécois incapable d'exécuter les exercices que lui commandent les officiers recruteurs; habile aux travaux des champs, il n'entend rien aux manœuvres militaires et finit par décourager les recruteurs:

"Baptiste, que m'dit l'z'officier, t'es mieux d'aller travailler dans tes champs. T'es déchargé, tu feras jamais un soldat...» - «Ben, j'vas dire là, toé que j'y 
répercure, j'aime mieux rester habitant que d'être soldat pis faire des singeries avec des fusils qui sont tant seurement pas capables de quer une mouche! Pis r'viens pas me badrer cheu nous, toé, parce que j'en ai un fusil moi itou, pis j't'avartis qu'y est chargé c'lui-là!” Là-dessus, j'ai pris le bord. Quand les criatures m'ont vu arriver, vous parlez qu'y m'ont fait une fête! Faut vous dire qu'y avait pus rien que moé pis mossieu le curé dans la paroisse en fait d'hommes, les autres ont été conscriptionnés ou ben y sont morts ${ }^{6}$.

Le monologue ironise adroitement sur l'habileté du paysan québécois pour les travaux agricoles et sur son indifférence aux manœuvres militaires; il valorise les travaux civils au détriment de l'esprit guerrier, qu'il ridiculise. Son auteur, Armand Leclaire, était comédien et dramaturge. Il travaillait dans les "scopes" avec les bonimenteurs Alex Silvio, Hector Pellerin et autres. Son texte a été récité dans les théâtres, et a également été publié dans Le Passe-temps, magazine populaire de chanson et de musique largement répandu. La lecture que fait Leclaire de la conscription et du travail des recruteurs était certainement partagée par une grande partie de l'auditoire francophone de Montréal, lequel devait trouver assez ridicules les très nombreux films montrant les soldats à l'entraînement. Le public avait évolué un peu comme Leclaire, qui avait aussi écrit quelques années plus tôt une pièce protestant contre la suppression du français dans les écoles de l'Ontario.

Ces monologues et ces chansons, présentés dans les salles mêmes où étaient projetés les films officiels, constituaient peutêtre le seul commentaire réaliste de la guerre. Lus et entendus dans les «scopes" ou ailleurs, dits et chantés avant ou après les films, ils ne pouvaient manquer de constituer un contraste frappant. Le censeur canadien, Ernest J. Chambers, a d'ailleurs trouvé assez tôt raison de s'en plaindre. Son rapport insiste particulièrement sur les «numéros" et les pièces de théâtre:

[...] des hommes haut placés et de jugement [...] ont fait remarquer que les chansons de certains vaudevilles exprimaient une envie pathétique de la paix à tout prix et visaient de toute évidence à provoquer un sentiment de lassitude à l'égard de la guerre (p. 276). 
La répression de ces textes résistants fut faite par l'entremise des critiques dramatiques des journaux, auxquels le censeur demanda de décourager la présentation de tels spectacles. Lorsque les représentations suspectes se poursuivaient, les renseignements fournis par les journaux étaient transmis à la police qui intervenait, mais une partie des textes réfractaires échappait probablement au censeur, parce que le public de gens "haut placés et de jugement » ne fréquentait pas beaucoup les salles de vaudeville, et les critiques dramatiques des journaux en parlaient assez rarement. Il est donc permis de penser que la résistance à la propagande pouvait se manifester dans le spectacle populaire; elle était probablement allusive plutôt qu'explicite, mais elle était lisible pour qui avait appris cette sorte de lecture.

Au Québec, le cinéma oral est demeuré une pratique en vogue jusqu'à l'arrivée du cinéma parlant, mais par la suite, d'autres pratiques résistantes sont apparues, venant contrer ou atténuer les pratiques hégémoniques. Aussi bâtardes que le cinéma oral, ces pratiques sont le théâtre cinématographique, la radio visuelle et le cinéma radiophonique, qui sont trois formes d'appropriation locale du cinéma étranger, trois formes de domestication du "fantôme de la vérité" par des strates différentes de la couche dominante.

\section{Le théâtre cinématographique}

Le théâtre cinématographique consiste dans l'adaptation au théâtre de sujets populaires au cinéma. Au Québec, cette pratique est apparue vers 1920 ; assez marginale, elle a quand même produit un bon nombre de textes dont certains ont connu un très grand succès sur scène. La première de ces pièces est probablement la revue Allo Chat-Plin. Présentée en décembre 1915, cette comédie en trois actes qui tenait autant de la pièce que de la revue portait sur l'actualité locale. Charlot en était le personnage principal; c'est lui qui assurait le lien entre les tableaux ${ }^{7}$. C'est dire qu'au centre de la narration, on trouve un personnage de cinéma. L'adaptation suivante semble avoir été Les Dopés, montée en 1918 par Paul Gury, un Breton vivant au Québec, qui a écrit de nombreux mélodrames théâtraux entre 1930 et 1950 et qui a dirigé deux films québécois des années 
cinquante ${ }^{8}$. Cette pièce avait probablement été adaptée des nombreux films sur le sujet produits après la guerre?. Gury a aussi écrit Les Esclaves blanches, peut-être adaptée du populaire film danois réalisé par Alfred Lind en 1911, La Traite des Blanches (Den Hvide Slavinde), ou du moins inspiré des mêmes récits et des mêmes thèmes: enlèvements de femmes, prostitution, criminalité et exploitation. Le film danois avait eu tant de succès aux États-Unis qu'une adaptation en a été faite en 1913 par George Loane Tucker pour Universal: Traffic in Souls (Sadoul, p. 350).

L'exemple le mieux connu, et qui a attiré le public et la critique plus que tout autre, est Le Cheik ou Entre deux civilisations, écrit en 1923 par Armand Leclaire. La pièce reposait de toute évidence sur les éléments qui avaient fait le succès du film The Sheik (Georges Melford, 1921) avec Rudolf Valentino, mais aussi sur l'immense popularité dont l'acteur jouissait alors. La pièce raconte les aventures d'un groupe de Québécois qui rencontrent des Arabes dans le désert nord-africain, où le fils d'un cheik tente de séduire la fille d'un géologue qu'il a rencontrée auparavant à Montréal. La pièce est assez semblable au film, histoire raciste d'une jeune Anglaise tombée amoureuse du cheik Ahmed, ensuite enlevée par un bandit, plus tard sauvée par le cheik qui révèle alors son origine blanche et peut épouser l'héroïne. L'histoire d'amour est centrale dans la pièce comme dans le film, et elle suscite une intrigue dynamique où les péripéties se succèdent à un rythme enlevant. Selon l'historien Alonzo Leblanc, cette pièce a de toute évidence été créée pour exploiter le succès du film de Valentino; et sa forme mélodramatique la destinait certainement au public populaire des cinémas. La pièce a été présentée au thêâtre Chanteclerc à Montréal (p. 441-442). Elle a obtenu un succès considérable et a été jouée à maintes reprises au cours des années suivantes.

Les mêmes films mettant en vedette Valentino ont inspiré un autre auteur, dont ces adaptations seront la spécialité. Pendant une carrière de deux décennies, il a écrit plusieurs pièces explicitement adaptées de films américains. Son nom était Ernest Guimond, mais il écrivait aussi sous le pseudonyme de Jean Bart, ce qui n'était certainement pas fortuit, Jean Bart étant un 
pirate français. En 1927, Guimond a profité, lui aussi, de la grande popularité des histoires arabes pour écrire une pièce sur le sujet. Probablement assez proche de la pièce de Leclaire, la sienne s'intitulait Le Fils du cheik et racontait le conflit entre un cheik et son fils au sujet de l'amoureuse blanche de ce dernier (Rinfret, p. 147). La pièce semble aussi avoir été une adaptation assez fidèle du dernier film de Valentino Son of the Sheik (George Fitzmaurice, 1926).

Guimond voulait évidemment profiter de la popularité de Valentino et des autres pièces inspirées par ces films. Or, il avait déjà écrit des textes semblables, le premier encore inspiré d'un autre film de Georges Fitzmaurice, qui avait réalisé Son of the Sheik. Né en France, Fitzmaurice avait aussi été directeur de théâtre; ses films étaient peut-être d'une facture plus théâtrale et plus facile à adapter pour la scène. Sous la plume de Guimond, son film The Cheat (1923) est devenu une pièce intitulée La Marque d'infamie, écrite en 1924 et clairement inspirée du film, de l'aveu même de Guimond (Rinfret, p. 152). La pièce a été montée la même année. Le texte original a été perdu et les descriptions en sont vagues, mais le film lui-même avait eu une histoire répétitive. Un premier film avait été fait en 1915 par Cecil B. de Mille, et une autre version avait été réalisée en 1923 par Fitzmaurice, avec Pola Negri (Sadoul, p. 163).

The Cheat raconte une autre histoire raciste, celle d'une riche famille américaine menacée par la présence d'un voisin asiatique qui convoite la femme du héros. Lorsque celle-ci est ruinée par un mauvais placement, l'Asiatique propose de lui donner de l'argent si elle accepte de faire l'amour avec lui. Comme elle refuse, il la marque avec un fer brûlant. Le mari est traîné en cour après avoir tué le traître, mais il est disculpé lorsque sa femme montre la marque. Ce film eut un succès retentissant en France grâce à son style soigné et inventif (utilisant beaucoup le gros plan et dosant habilement la lumière et l'ombre). Au Québec, c'est probablement son inspiration mélodramatique qui lui valut un grand succès.

En 1928, Guimond a écrit Le Sinistre Fantôme (Rinfret, p. 159), imitant le célèbre Phantom of the Opera (Rupert Julian, 1925). La pièce a été présentée en 1928 au Chanteclerc, théâtre 
alors dirigé par Alex Silvio, surnommé à Montréal «le roi des conférenciers", mais qui semble aussi avoir été le roi des producteurs de pièces adaptées de films. L'histoire est aujourd'hui bien connue, mais il importe d'en rappeler l'intrigue parce que c'est probablement sa teneur mélodramatique qui donne la raison de son adaptation au Québec. Un homme défiguré vit secrètement dans les sous-sols de l'Opéra de Paris, où il tombe amoureux d'une jeune étudiante en musique. Il essaie de la garder prisonnière de son souterrain et de se faire aimer d'elle, mais il est tué par les gens venus à la recherche de la jeune fille. De toute évidence, les Québécois aimaient aussi les histoires d'enlèvement. Cela a-t-il un rapport avec la captivité nationale? La métaphore est facile, mais on constate qu'elle revient dans plusieurs de ces mélodrames adaptés; elle pourrait aussi désigner la rigoureuse morale imposée alors par un clergé puissant et puritain. De nombreux Québécois, hommes ou femmes, pouvaient alors rêver d'être enlevés par un personnage mystérieux, ou d'enlever un amant ou une amante malgré une possible fin tragique... Le fantôme de l'opéra est donc devenu celui de la vérité en proposant à l'imaginaire local un récit étranger qui transgressait une situation politique et une morale contraignantes.

Cette adaptation identitaire par transfert intermédiatique semble avoir été une tactique fort populaire. En 1926, Guimond a écrit La Vierge blanche, inspirée de toute évidence du film White Sister (Henry King, 1923) tourné en Italie avec Lillian Gish et Ronald Colman. Ce film racontait l'amour déçu d'un officier italien pour une jeune couventine. L'officier est déclaré mort par la sœur jalouse de la couventine, qui entre alors chez les sœurs blanches. Parti puis revenu, l'officier désire obtenir du pape la dispense qui lui permettrait d'épouser la religieuse. Mais le Vésuve fait alors éruption et l'officier est tué en portant secours aux victimes; la religieuse retourne alors au "service de Dieu" (Connelly, p. 306). Notons que le film White Sister est lui-même adapté d'une nouvelle écrite en 1909 par F. Marion Crawford; l'auteur a aussi écrit une pièce, et une première version du film avait été faite en 1915 par Fred Wright pour Essanay (King Hanson, p. 1028). Dans cette version, l'officier était enlevé par des «sauvages" plutôt que déclaré mort 
par la sœur jalouse. La pièce de Guimond, qui a été présentée au Chanteclerc et au théâtre Saint-Denis, semble avoir été assez semblable au film. Mais le plus étonnant, c'est que deux autres adaptations du même film virent le jour au Québec. L'auteur dramatique Marc Forrez a écrit un mélodrame en quatre parties sous le titre Vengeance d'amour ou Sxur blanche, dont la source était explicite (Rinfret, p. 44). Une autre pièce portant le même titre a aussi été écrite par un auteur nommé Charles E. Harpe, probablement un pseudonyme de Maurice Beaupré, acteur et dramaturge occasionnel (p. 191) ${ }^{10}$. Le texte en a été conservé et ressemble beaucoup à l'histoire du film: une sœur jalouse complote pour éloigner l'amant qui est ensuite supposé mort. La sœur éplorée décide de devenir religieuse; son amoureux s'enrôle dans l'aviation, mais blessé à mort, meurt dans ses bras. Ce récit fut aussi populaire que celui du Cheik, probablement parce qu'il permettait d'expérimenter sur le plan symbolique des affects interdits par une société très policée. Ce transfert intermédiatique permettait d'ailleurs la diffusion de ces récits venus du cinéma sans subir les foudres d'un clergé qui avait fait du film son pire ennemi.

\section{La radio visuelle}

La radio visuelle est une autre pratique intermédiatique qui consiste en l'adaptation radiophonique d'œuvres cinématographiques. Elle a été pratiquée de façon systématique et explicite pendant plusieurs années, principalement par l'entremise d'une émission appelée Radio-Théâtre Lux Français, qui semble avoir imité la formule américaine du Lux Radio Theater, lequel présentait à la radio des États-Unis des versions adaptées des films les plus populaires (Thomas, 1991) ${ }^{11}$. L'émission québécoise a été présentée entre 1942 et 1945 à CKAC et CHRC, et reposait sur des pièces adaptées du cinéma ou du théâtre (Pagé, 1975, p. 662). Henry Deyglun, qui a beaucoup écrit pour la scène, a également adapté plusieurs récits pour le Théatre Lux (Legris, p. 89). Paul L'Anglais, le producteur de cette émission (p. 57), avait auparavant été chanteur à la radio (Proulx, p. 34) et avait écrit pour la radio les feuilletons Jeanne et Arthur et Ceux quon aime, traduits de radio-romans américains (Pagé, 1975, p. 353). Une émission 
semblable a été diffusée par CBF en 1942 et 1943, intitulée Silver Theater ou Théâtre d'Hollywood (p. 662). D'autres émissions se sont inspirées de cette veine bien que de façon moins explicite. La radio fit rapidement appel à un grand nombre d'auteurs, qui devaient beaucoup produire pour suffire à la demande. Ernest Guimond, qui avait écrit des pièces de théâtre adaptées de films américains, a également écrit plusieurs pièces radiophoniques sous le même pseudonyme de Jean Bart (Legris, p. 89).

Le plus digne représentant de cette radio visuelle est peut-être Henri Letondal. "Scripteur" de radio des plus prolifiques, il avait d'abord été chroniqueur de cinéma et de théâtre pour le journal La Parrie (de 1921 à 1925) ainsi que comédien et chanteur à la station CKAC. Pendant les années trente il a été l'animateur du cabaret Le Matou botté, où il montait des revues et interprétait ses propres chansons, et a écrit pour la radio des dizaines de pièces dont certaines, comme Meurtre au studio ou La Photo révélatrice, semblent exploiter les mêmes thèmes que le cinéma populaire: Le Supplice indien, La Mystérieuse Dame blonde, Le Pèlerin de Bénarès, etc. Il a ensuite passé de nombreuses années (de 1939 à 1955) à Hollywood où il a joué dans une trentaine de films et a réalisé pour CKAC l'émission Hollywood vous parle (Pagé, 1978, p. 13-14 et 43). Cette émission n'était pas la seule qui était consacrée au cinéma puisque CKAC présentait aussi, trois fois par semaine, entre 1938 et 1942, Radio-Cinéma Revue écrite par Jeanne Frey (Pagé, 1975, p. 293) Les autres stations produisaient probablement des émissions semblables, et le journal populaire Radiomonde vantait les vedettes du cinéma autant que les émissions de radio qui les faisaient connaître. L'écoute des enregistrements conservés permettrait sans doute de reconnaître la musique des films mentionnés dans les émissions, mais la production locale instaurait une instance d'énonciation fondant une subjectivité hybride plutôt que fondée uniquement sur le rapport à l'extérieur.

\section{Le cinéma radiophonique}

L'étape ultime de ce processus a été ce qu'on pourrait appeler le "cinéma radiophonique": l'adaptation cinématographique des œuvres de fiction les plus populaires de la radio. Après la 
production du Père Chopin (1944), scénarisé par l'auteur de radio Jean Desprez, le financier René Germain a vite compris combien les œuvres radiophoniques pouvaient être rentables; aussi a-t-il requis les services du producteur de radio Paul L'Anglais (Bonneville, p. 5). Trois des cinq films - Un homme et son péché (1949), Le Curé de village (1949), Séraphin (1950) -, que ce dernier a dirigés à titre de producteur de cinéma, ont donc été tirés du répertoire radiophonique et ont été réalisés par ce vieux routier de la radio qu'était Paul Gury. En outre, les trois films ont été joués en grande partie par les comédiens qui avaient popularisé les personnages à la radio: Hector Charland, Ovila Légaré, Nicole Germain, etc. (Lever, p. 101), les voix de ces comédiens ne pouvant manquer de rappeler aux spectateurs québécois les émissions de radio où ils les avaient d'abord entendues. Paul L'Anglais s'est d'ailleurs plaint de cette distribution trop radiophonique, les comédiens ignorant le jeu du cinéma (Bonneville, p. 9). L'Anglais a néanmoins produit en 1952 le Tit-Coq de Gratien Gélinas, auteur qui avait acquis sa renommée à la radio grâce à son célèbre personnage Fridolin (Pagé, 1976, p. 81). Le cinéma national contribuait ainsi à singulariser et à consolider par emprunt intermédiatique une culture hybride largement fondée sur l'adaptation, donc aussi sur le déplacement de l'instance de validation des discours.

\section{Médias et territoires}

Rappelant les hypothèses de Derrida sur une graphie précédant toute oralité, Deleuze et Guattari supposent également que la représentation territoriale est faite de deux éléments hétérogènes: voix et graphisme. L'apparition et l'évolution du "socius" provoquent "[...] la grande tâche du refoulement germinal intense. Ce qui est refoulé, en effet, c'est le corps plein comme fond de la terre intense, qui doit faire place au socius en extension dans lequel passent ou ne passent pas les intensités en cause" $(1974$, p. 241). Selon eux, l'extension du social crée un régime de connotation où la chose elle-même devient signe, à cause d'un graphisme connoté à la voix. Le déséquilibre des deux éléments est rattrapé par l'œil qui voit le mot "[...] pour autant qu'il évalue la douleur du graphisme" (p. 241). 
Ce traumatisme lié au développement de la graphie serait aussi le corollaire de l'apparition et du développement des États. "La pseudo-territorialité est le produit d'une effective déterritorialisation qui substitue des signes abstraits aux signes de la terre, et qui fait de la terre elle-même une propriété d'État, ou de ses plus riches serviteurs et fonctionnaires" (p. 232). La machine despocique d'État surcode les flux territoriaux, mais ceux-ci échappent, refluent, se décodent et débordent sur d'autres territoires et dans d'autres canaux. Cette description de la naissance du rapport entre le signe et l'État semble radicale, mais elle est pourtant assez semblable à celle des rapports entre de nouveaux médias. D'abord espaces vides où s'engouffrent les flux décodés, ils sont rapidement encerclés et quadrillés par la machine bureaucratique qui y impose le surcodage, mais ils ont tout de même constitué des territoires sauvages, des "espaces lisses " affirment Deleuze et Guattari (1980), c'est-à-dire des lieux d'expression non encore soumis à l'institution, lieux où se sont précipités les flux décodés avant que la machine despotique n'en contrôle toutes les entrées.

L'observation permet également de constater que la sphère intermédiatique suscite la constitution de territoires symboliques qui compensent la réduction de l'espace social et politique. La sphère intermédiatique pourrait donc être envisagée comme un espace temporairement nomade où l'apparition de nouveaux médias non encore arraisonnés permettrait à certaines communautés d'occuper un terrain symbolique leur échappant dans l'espace géo-politique. Le fantôme de la vérité, loin d'être un spectre, serait l'instance indispensable supposée par Berrendonner, et correspondrait aussi à ce que Ducrot appelle polyphonie: un point de vue qui paraît s'imposer à un certain niveau d'analyse (1980, p. 38-42). Au Québec la communauté francophone suscita ainsi un contexte de validation de son affirmation culturelle en adaptant à de nouveaux médias non encore institutionnalisés les récits hégémoniques produits à l'étranger.

L'intermédialité est certainement un phénomène contemporain, du moins sur son versant technologique, où les prolongements matériels des sens provoquent une prolifération exponentielle et une virtualisation des espaces pluritopiques et des 
configurations déictiques. Cette prolifération peut fournir le modèle permettant d'explorer à rebours les configurations antérieures, et ensuite par anticipation, celles qu'on peut projeter en examinant la généalogie ainsi retracée. Il en ressort que se développent concurremment deux sortes de configurations déictiques: une'strate plus englobante dont dépend la structure dominante des sociétés, et des strates inférieures qui ne s'y rattachent qu'épisodiquement, qui peuvent même s'en détacher ou y résister dans les cas où les intérêts deviennent trop antagoniques.

Analysée selon ce modèle, la nouvelle sphère intermédiatique offre certainement un terrain pour le développement d'une oligarchie mondiale aux tentacules effrayantes. Elle laisse aussi supposer l'apparition d'autres forces, d'autres réseaux, et la compréhension des rapports de forces dans ces réseaux ne peut probablement plus être appelée faire la ligne, pas plus que faire le point, et peut-être ces expressions n'ont-elle jamais été appropriées. Déjà dans la préhistoire, quand les nomades ont commencé à occuper des territoires distants en effectuant des migrations saisonnières, il fallait peut-être trouver une expression plus adéquate que faire la ligne, car il ne s'agit plus seulement de la médiane entre deux plans, mais de plusieurs lignes reliant des surfaces distantes. Peut-être fallait-il déjà parler de faire la trame, ou même de faire le net?

Université Laval

\section{NOTES}

I Cette expression est empruntée au titre du premier colloque du CRI : La nouvelle sphère intermédiatique.

2 Voir à ce sujet le livre de Gilles Deleuze, Nietzsche et la philosophie (Paris: P.U.F., 1983, p. 44-82).

3 Voir à ce sujet notre thèse de doctorat, Le Bonimenteur et le cinéma oral. Les vues animées entre tradition et modernité (Montréal: Université de Montréal, 1996).

4 Voir Gilles Deleuze, L'Image-temps (Paris: Minuit, 1985, p. 203-245). Voir aussi Bruno Alcala, "Philosopher avec le cinéma: Gilles Deleuze, temps et pensée " (CinémAction, n ${ }^{\circ} 47,1988$, p. 79 à 84).

5 Anonyme, "Une jolie soirée au Monument ", La Presse, 8 octobre 1915, p. 2.

6 Armand Leclaire, "Le conscrit Baptiste", Le Passe-temps, 8 septembre 1917.

7 Anonyme, “Dans nos théâtres. Allo Chat-Plin ", La Presse, 18 décembre 1915. 
8 Son vrai nom était Loic Le Guriadec. Il dirigea Un homme et son péché en 1949 et Séraphin en 1950, deux adaptations d'un roman à succès de Claude-Henri Grignon. Il réalisa aussi en 1949 Le Curé de village. Voir à ce sujet Pierre Véronneau, Cinéma de l'époque duplessiste (Montréal : Cinémathèque québécoise, 1979).

9 Au Québec, cette phobie mena même à la production d'un film, probablement inspiré par Hollywood, La Drogue fatale (J. A. Homier, 1924), produit après la présentation à Montréal d'un film américain intitulé La Pire Menace.

10 Le manuscrit, conservé par la Bibliothèque nationale du Québec, porte les informations suivantes: Sacur Blanche, par Charles E. Harpe. Tous droits réservés par Maurice Beaupré, 1942.

11 Outre le Lux Radio Theatre, l'auteur nomme The Screen Guild Theatre, autre émission de radio adaptée des films hollywoodiens.

\section{OUVRAGES CITÉS}

Alcala, Bruno. "Philosopher avec le cinéma: Gilles Deleuze, temps et pensée". CinémAction, $\mathrm{n}^{\circ} 47$ (1988), p. 79-84.

Berrendonner, Alain. Éléments de pragmatique linguistique. Paris: Minuit, 1981.

Bonneville, Léo. "Rencontre avec Paul L’Anglais". Séquences, n" 106 (1981), p. 5-9.

Chambers, Ernest, "Rapport sur le service de la censure de la presse canadienne». Cahiers d'histoire polirique, $\mathrm{n}^{\circ} 2$ (1996), p. 181-288.

Connelly, Robert. The Motion Picture Guide. Silent Film 1910-1936. Chicago: Cinebooks, 1986.

Deleuze, Gilles. Nietzsche et la philosophie. Paris : P.U.F., 1983.

Deleuze, Gilles. L'Image-temps. Paris: Minuit, 1985.

Deleuze, Gilles et Félix Guattari. L'Anti-CEdipe. Paris: Minuit, 1974.

Deleuze, Gilles et Félix Guattari. Mille Plateaux. Paris : Minuit, 1980.

Deleuze, Gilles et Claire Parnet. Dialogues. Paris : Flammarion, 1977.

Ducrot, Oswald. Dire et ne pas dire. Paris: Hermann, 1972.

Ducrot, Oswald. Les Mots du discours. Paris: Minuit, 1980.

Godzich, Wlad et Jeff Kittay. The Emergence of Prose. An Essay in Prosaics. Minneapolis: University of Minnesota Press, 1987.

Hébert, Chantal. Le Burlesque au Québec. Un divertissement populaire. Montréal: Hurtubise HMH, 1981.

King Hanson, Patricia, (direction). The American Film Institute Catalog. Feature Films 1911 1920. Berkeley: University of Los Angeles Press, 1988.

Lacasse, Germain. Le Bonimenteur et le cinéma oral. Les vues animées entre tradition et modernité (thèse de doctorat). Montréal: Université de Montréal, 1996.

LeBlanc, Alonzo. "Entre deux civilisations, drame d'Armand Leclaire", dans Maurice Lemire (direction), Dictionnaire des euvres littéraires du Québec, tome II 1900-1939. Montréal : Fides (1978), p. 441-442.

Legris, Renée. Dictionnaire des auteurs du radio-feuilleton québécois. Montréal: Fides, 1981.

Leroi-Gourhan, André. Le Geste et la parole. Technique et langage. Paris: Albin-Michel, 1965.

Lever, Yves. Histoire générale du cinéma au Québec. Montréal : Boréal, 1988.

McLuhan, Marshall. Pour comprendre les médias. Les prolongements technologiques de l'homme. Montréal: Hurtubise-HMH, 1972. 
Rinfret, Edouard. Le Théatre canadien d'expression française, tome 2. Montréal: Leméac, 1976.

Pagé, Pierre. Répertoire des auvres de la littérature radiophonique québécoise 1930-1970. Montréal : Fides, 1975.

Pagé, Pierre. Le Comique et l'humour à la radio québécoise, vol. 1. Montréal : Éditions La Presse, 1976.

Pagé, Pierre. Le Comique et l'humour à la radio québécoise, vol. 2. Montréal : Éditions La Presse, 1978.

Proulx, Gilles. L'Aventure de la radio au Québec. Montréal : Éditions La Presse, 1978.

Sadoul, Georges. Histoire générale du cinéma, tome 3. Le cinéma devient un art. Paris: Denoël, 1975.

Thomas, François, «Radio et cinéma». Autrement, n 9 (1991), p. 193-194.

Véronneau, Pierre. Cinéma de l'époque duplessiste. Montréal: Cinémathèque québécoise, 1979. 